\title{
Asymptotic behavior of positive solutions to a nonlinear biharmonic equation near isolated singularities
}

\author{
Hui Yang* \\ Yau Mathematical Sciences Center, Tsinghua University, Beijing 100084, China
}

\begin{abstract}
In this paper, we consider the asymptotic behavior of positive solutions of the biharmonic equation

$$
\Delta^{2} u=u^{p} \quad \text { in } B_{1} \backslash\{0\}
$$

with an isolated singularity, where the punctured ball $B_{1} \backslash\{0\} \subset \mathbb{R}^{n}$ with $n \geq 5$ and $\frac{n}{n-4}<p<\frac{n+4}{n-4}$. This equation is relevant for the $Q$-curvature problem in conformal geometry. We classify isolated singularities of positive solutions and describe the asymptotic behavior of positive singular solutions without the sign assumption for $-\Delta u$. We also give a new method to prove removable singularity theorem for nonlinear higher order equations.
\end{abstract}

Key words: Biharmonic equations, isolated singularities, asymptotic behavior, positive singular solutions.

Mathematics Subject Classification (2010): 35J30; 35B40; 35 B65

\section{Introduction and main results}

In this paper, we study the asymptotic behavior of positive solutions of the biharmonic equation

$$
\Delta^{2} u=u^{p} \quad \text { in } B_{1} \backslash\{0\}
$$

with an isolated singularity, where the punctured ball $B_{1} \backslash\{0\} \subset \mathbb{R}^{n}$ with $n \geq 5$ and $\frac{n}{n-4}<p<\frac{n+4}{n-4}$. Here the unit ball $B_{1}$ can be replaced by any bounded domain $\Omega \subset \mathbb{R}^{n}$ containing 0 . This equation serves as a basic model of nonlinear fourth-order equations and is also related to the $Q$-curvature problem in conformal geometry. Equation 1.1. and related equations arise in several models describing various phenomena

*E-mail addresses: mahuiyang@ust.hk; hui-yang15@outlook.com 
in the applied sciences see, for instance, Gazzola, Grunau and Sweers [13]. For an introduction to the $Q$-curvature problem see, for instance, Hang and Yang [20].

We first recall that the corresponding second order equation (when $n \geq 3$ and $\left.\frac{n}{n-2}<p<\frac{n+2}{n-2}\right)$

$$
-\Delta u=u^{p} \quad \text { in } B_{1} \backslash\{0\}
$$

was studied by Gidas-Spruck [15] and Caffarelli-Gidas-Spruck [3]. More specifically, the following classification result is obtained.

Theorem A ( [3, 15]) Let $n \geq 3$ and $u \in C^{2}\left(B_{1} \backslash\{0\}\right)$ be a positive solution of [1.2). Assume

$$
\frac{n}{n-2}<p<\frac{n+2}{n-2}
$$

Then either the singularity at $x=0$ is removable, or $u$ is a distribution solution in the entire ball $B_{1}$, and

$$
\lim _{|x| \rightarrow 0}|x|^{\frac{2}{p-1}} u(x)=C_{0},
$$

where

$$
C_{0}=\left\{\frac{2(n-2)}{(p-1)^{2}}\left(p-\frac{n}{n-2}\right)\right\}^{\frac{1}{p-1}}
$$

In addition, the asymptotic behavior of positive solutions of (1.2) near an isolated singularity was studied by Lions [25] for $1<p<\frac{n}{n-2}$, by Aviles [1] for $p=\frac{n}{n-2}$, by Caffarelli-Gidas-Spruck [3] and by Korevaar-Mazzeo-Pacard-Schoen [22] in the case $p=\frac{n+2}{n-2}$ and by Bidaut-Véron and Véron [2] when $p>\frac{n+2}{n-2}$. Hence the isolated singularities of positive solutions for the second order equation (1.2) have been very well understood. The asymptotic behavior of positive solutions for a more general second order equation $-\Delta u=K(x) u^{\frac{n+2}{n-2}}$ with isolated singularity was studied by Chen-Lin [7, 8] and Taliaferro-Zhang [30]. See also González [16], Li [23] and HanLi-Teixeira [19] for a fully nonlinear equation of second order.

In the fundamental paper [24], Lin classified all positive smooth entire solutions of 1.1) with $1<p \leq \frac{n+4}{n-4}$ in $\mathbb{R}^{n}$ via the moving plane method. We refer to ChangYang [6], Martinazzi [26] and Wei-Xu [31] for the classification of smooth solutions of the higher-order equations in $\mathbb{R}^{n}$. For the supercritical case, that is for $p>\frac{n+4}{n-4}$, the positive smooth radial symmetric solutions of 1.1 in $\mathbb{R}^{n}$ were studied by GazzolaGrunau [12], Guo-Wei [17] and Winkler [32]. We also refer to a recent paper FrankKönig [11] for a classification of positive singular solutions to (1.1) with $p=\frac{n+4}{n-4}$ in $\mathbb{R}^{n} \backslash\{0\}$, where the positive singular solutions are radially symmetric about the origin (see Theorem 4.2 in [24]).

As far as we know, the classification of isolated singularities of positive solutions and the asymptotic behavior of positive singular solutions to fourth order equation (1.1) in $B_{1} \backslash\{0\}$ are far less known than the second order problem (1.2). Remark that, a positive solution $u$ of (1.1) in $B_{1} \backslash\{0\}$ may not be radially symmetric. 
If one looks closely at the tools being used in the proofs of second order problems, then one finds that the maximum principle plays an essential role. This is a crucial distinction from higher order problems for which there is no the maximum principle. Here and in the sequel "higher order" means order at least four. Another important tool intensively used for second order problems is the truncation method. This method is powerful in regularity theory and in properties of first order Sobolev spaces. However, the truncation method also fails for higher order problems. Therefore, the methods of above mentioned papers for second order problems cannot be applied to the fourth order equation 1.1.

Nevertheless we succeed here in proving exact asymptotic behavior of positive singular solutions for (1.1) which is completely analogous to its second order counterpart. Remark that our proof is very different from that of Theorem A in [3, 15]. Our main result is the following

Theorem 1.1. Let $n \geq 5$ and $u \in C^{4}\left(B_{1} \backslash\{0\}\right)$ be a nonnegative solution of (1.1). Assume

$$
\frac{n}{n-4}<p<\frac{n+4}{n-4} \text {. }
$$

Then either the singularity at $x=0$ is removable, or $u$ is a distribution solution in the entire ball $B_{1}$, and

$$
\lim _{|x| \rightarrow 0}|x|^{\frac{4}{p-1}} u(x)=C_{p, n}>0,
$$

where $C_{p, n}=\left[K_{0}(p, n)\right]^{\frac{1}{p-1}}$ and

$$
\begin{aligned}
K_{0}(p, n)= & \frac{8}{(p-1)^{4}}\left[(n-2)(n-4)(p-1)^{3}+2\left(n^{2}-10 n+20\right)(p-1)^{2}\right. \\
& -16(n-4)(p-1)+32] .
\end{aligned}
$$

Remark 1.1. We don't need any additional assumptions for $-\Delta u$ in $B_{1} \backslash\{0\}$ and for boundary conditions. Soranzo [29] studied the local behavior of positive solutions of (1.1) with additional assumption

$$
-\Delta u \geq 0 \quad \text { in } B_{1} \backslash\{0\} .
$$

Under the assumption [1.5], Soranzo [29] classified the isolated singularities of positive solutions of (1.1) for $1<p<\frac{n}{n-4}$ and obtained an upper bound of radially symmetric positive solutions of (1.1) for $p \geq \frac{n}{n-4}$. Theorem 1.1 also answers an open question raised in [29] (see Remark 5 there) and shows, in particular, that the nonnegativity of $-\Delta u$ in this problem is not necessary. Recently, Jin and Xiong [21] proved sharp blow up rates and the asymptotic radial symmetry of positive solutions of 1.11 with $p=\frac{n+4}{n-4}$ near the singularity under the sign assumption (1.5). We also mention that Ferrero-Grunau [10] have obtained the asymptotic behavior of positive radial singular solutions for biharmonic operator and power-like nonlinearity with the Dirichlet boundary condition. 
Remark 1.2. In $\mathbb{R}^{n}$, suppose $u$ is a positive smooth function satisfies equation (1.1) with $p>1$, then necessarily we have

$$
-\Delta u>0 \quad \text { in } \mathbb{R}^{n} .
$$

See Theorem 3.1 in Wei-Xu [31]. This important fact about $-\Delta$ u enables the maximum principle to be applied to positive solutions of 11.1 in $\mathbb{R}^{n}$. Such as see [24.31]. Hence the positive solutions of equation 1.1 in $\mathbb{R}^{n}$ provide enough information for applying the maximum principle, but this is not true for (1.1) in $B_{1} \backslash\{0\}$.

Remark 1.3. When $\frac{n}{n-4}<p<\frac{n+4}{n-4}$, it is well known that the function

$$
u(x)=C_{p, n}|x|^{-\frac{4}{p-1}}
$$

is an exact positive singular solution of (1.1) which obviously satisfies asymptotic behavior [1.3). See also Guo-Wei-Zhou [18] for another a family of positive singular radial solutions of (1.1) in $\mathbb{R}^{n} \backslash\{0\}$.

The rest of this paper is organized as follows. In Section 2, we establish some basic estimates. In Section 3, we prove Theorem 1.1 .

\section{Preliminaries}

In this section we establish some basic estimates. First we recall the following Liouville type theorem. For its proof, such as see Lin [24].

Theorem 2.1. ( [24]) Suppose that $u$ is a nonnegative solution of

$$
\Delta^{2} u=u^{p} \quad \text { in } \mathbb{R}^{n}
$$

for $1<p<\frac{n+4}{n-4}$. Then $u \equiv 0$ in $\mathbb{R}^{n}$.

By a doubling lemma of Polácik, Quittner and Souplet [28] and above Liouville theorem, we have the following singularity and decay estimates. Because their proof is similar, we only give the proof of decay estimates here.

Lemma 2.1. Let $u \in C^{4}\left(B_{1} \backslash\{0\}\right)$ be a nonnegative solution of (1.1) with $1<p<$ $\frac{n+4}{n-4}$. Then

$$
u(x) \leq C|x|^{-\frac{4}{p-1}} \quad \text { for }|x| \leq \frac{1}{2},
$$

where $C$ is a constant, depending on $n$ and $p$ only.

Remark 2.1. For the second order equation (1.2), if one has an upper estimate similar to (2.2), then one can easily obtain the following Harnack inequality

$$
\sup _{r \leq|x| \leq 2 r} u \leq C \inf _{r \leq|x| \leq 2 r} u,
$$

where $C$ is independent of $r$. Such as see [1,8,15,22]. This is an essential tool for these papers to study isolated singularities of second order problems. In a recent paper [4] 
Caffarelli, Jin, Sire and Xiong use a similar Harnack inequality to classify isolated singularities of positive solutions of a fractional equation. However, this Harnack inequality does not generally hold for fourth order equation (1.1). In particular, if we suppose additionally that

$$
-\Delta u \geq 0 \quad \text { in } B_{1} \backslash\{0\}
$$

then Caristi-Mitidieri [5] proved that the similar Harnack inequality still holds for fourth order equation 1.11).

Remark 2.2. We also remark that the condition (2.4) is necessary for the validity of the Harnack inequality to biharmonic equations as the following simple example shows: consider the function $u(x)=\sum_{i=1}^{n} x_{i}^{2}$. It is nonnegative, satisfies $\Delta^{2} u=0$ and $\Delta u=2 n$, but the Harnack inequality does not hold in $B_{1}(0)$.

Lemma 2.2. Let $u$ be a nonnegative solution of

$$
\Delta^{2} u=u^{p} \quad \text { in } B_{1}^{c},
$$

where $B_{1}^{c}:=\left\{x \in \mathbb{R}^{n}:|x|>1\right\}$. Assume $1<p<\frac{n+4}{n-4}$. Then

$$
u(x) \leq C|x|^{-\frac{4}{p-1}} \quad \text { for }|x|>2,
$$

where $C$ is a constant, depending on $n$ and $p$ only.

Proof. Suppose by contradiction that there exist a sequence of nonnegative solutions $\left(u_{k}\right)_{k}$ of (2.5) and a sequence of points $\left|x_{k}\right|>2$, such that

$$
M_{k}\left(x_{k}\right) d\left(x_{k}\right)>2 k, \quad k=1,2, \cdots,
$$

where $M_{k}(x):=\left(u_{k}(x)\right)^{\frac{p-1}{4}}$ and $d(x):=\operatorname{dist}\left(x, \partial B_{1}^{c}\right)=|x|-1$ for $x \in B_{1}^{c}$. By the doubling lemma of [28] there exists another sequence $y_{k} \in B_{1}^{c}$ such that

$$
M_{k}\left(y_{k}\right) d\left(y_{k}\right)>2 k, \quad M_{k}\left(y_{k}\right) \geq M_{k}\left(x_{k}\right)
$$

and

$$
M_{k}(z) \leq 2 M_{k}\left(y_{k}\right) \text { for any }\left|z-y_{k}\right| \leq k \lambda_{k} .
$$

where $\lambda_{k}:=M_{k}\left(y_{k}\right)^{-1}$. We now define

$$
\bar{u}_{k}(x)=\lambda_{k}^{\frac{4}{p-1}} u_{k}\left(y_{k}+\lambda_{k} x\right) \quad \text { for } x \in B_{k}(0) .
$$

Then $\bar{u}_{k}$ is a nonnegative solution of

$$
\Delta^{2} \bar{u}_{k}=\left(\bar{u}_{k}\right)^{p} \quad \text { in } B_{k}(0) .
$$

Moreover,

$$
\bar{u}_{k}(0)=1 \quad \text { and } \quad \max _{B_{k}(0)}\left|\bar{u}_{k}\right| \leq 2^{\frac{4}{p-1}} .
$$

By the elliptic estimates, we deduce that a subsequence of $\left(\bar{u}_{k}\right)_{k}$ converges in $C_{\text {loc }}^{4}\left(\mathbb{R}^{n}\right)$ to a nonnegative solution $u_{\infty}$ of $(2.1)$ in $\mathbb{R}^{n}$. By (2.7), we have $u_{\infty}(0)=1$. This contradicts Theorem 2.1 
Corollary 2.1. Let $u \in C^{4}\left(B_{1} \backslash\{0\}\right)$ be a nonnegative solution of (1.1) with $1<p<$ $\frac{n+4}{n-4}$. Then there exists a constant $C_{1}=C_{1}(n, p)$ such that for all $|x| \leq \frac{1}{4}$,

$$
\sum_{k \leq 3}|x|^{\frac{4}{p-1}+k}\left|\nabla^{k} u(x)\right| \leq C_{1}
$$

Proof. For any $x_{0}$ with $\left|x_{0}\right| \leq \frac{1}{4}$, take $\lambda=\frac{\left|x_{0}\right|}{2}$ and define

$$
\bar{u}(x)=\lambda^{\frac{4}{p-1}} u\left(x_{0}+\lambda x\right) .
$$

Then $\bar{u}$ is a nonnegative solution of 1.10 in $B_{1}$. By the Lemma 2.1, $|\bar{u}| \leq C_{2}$ in $B_{1}$. The standard elliptic estimates give

$$
\sum_{k \leq 3}\left|\nabla^{k} \bar{u}(0)\right| \leq C_{3}
$$

Rescaling back we obtain 2.9.

Using a similar scaling argument as above, we also have

Corollary 2.2. Let u be a nonnegative solution of 2.5 with $1<p<\frac{n+4}{n-4}$. Then there exists a constant $C_{2}=C_{2}(n, p)$ such that for all $|x| \geq 4$,

$$
\sum_{k \leq 3}|x|^{\frac{4}{p-1}+k}\left|\nabla^{k} u(x)\right| \leq C_{2}
$$

\section{Proof of the main result}

In this section we will prove Theorem 1.1. We first show that any nonnegative solution of 1.1) with $p \geq \frac{n}{n-4}$ is a solution in $B_{1}$ in the sense of distribution.

Lemma 3.1. Assume $p \geq \frac{n}{n-4}$ and that $u \in C^{4}\left(B_{1} \backslash\{0\}\right)$ is a nonnegative solution of (1.1). Then $u \in L_{l o c}^{p}\left(B_{1}\right)$ and $u$ is a distribution solution of (1.1) in $B_{1}$, that is,

$$
\int_{B_{1}} u \Delta^{2} \varphi=\int_{B_{1}} u^{p} \varphi \quad \text { for all } \varphi \in C_{c}^{\infty}\left(B_{1}\right) .
$$

Proof. For any $0<\epsilon \ll 1$, we take $\eta_{\epsilon} \in C^{\infty}\left(\mathbb{R}^{n}\right)$ with values in $[0,1]$ satisfying

$$
\eta_{\epsilon}(x)= \begin{cases}0 & \text { for }|x| \leq \epsilon \\ 1 & \text { for }|x| \geq 2 \epsilon\end{cases}
$$

and

$$
\left|\nabla^{k} \eta_{\epsilon}(x)\right| \leq C \epsilon^{-k} \quad \text { for } k=1,2,3,4 .
$$

Let $m=\frac{4 p}{p-1}$ and define $\xi_{\epsilon}=\left(\eta_{\epsilon}\right)^{m}$. Multiplying 1.1p by $\xi_{\epsilon}$ and integrating by parts in $B_{r}$ with $\frac{1}{2}<r<1$, we get

$$
\int_{B_{r}} u^{p} \xi_{\epsilon}=\int_{\partial B_{r}} \frac{\partial}{\partial \nu} \Delta u+\int_{B_{r}} u \Delta^{2} \xi_{\epsilon} .
$$


Note that

$$
\left|\Delta^{2} \xi_{\epsilon}\right| \leq C \epsilon^{-4}\left(\eta_{\epsilon}\right)^{m-4} \chi_{\{\epsilon \leq|x| \leq 2 \epsilon\}}=C \epsilon^{-4}\left(\xi_{\epsilon}\right)^{1 / p} \chi_{\{\epsilon \leq|x| \leq 2 \epsilon\}} .
$$

By Hölder's inequality, we obtain

$$
\begin{aligned}
\left|\int_{B_{r}} u \Delta^{2} \xi_{\epsilon}\right| & \leq C \epsilon^{-4} \int_{\{\epsilon \leq|x| \leq 2 \epsilon\}} u\left(\xi_{\epsilon}\right)^{1 / p} \\
& \leq C \epsilon^{-4} \cdot \epsilon^{n(1-1 / p)}\left(\int_{\{\epsilon \leq|x| \leq 2 \epsilon\}} u^{p} \xi_{\epsilon}\right)^{1 / p} \\
& \leq C\left(\int_{\{\epsilon \leq|x| \leq 2 \epsilon\}} u^{p} \xi_{\epsilon}\right)^{1 / p} .
\end{aligned}
$$

Hence we have

$$
\int_{B_{r}} u^{p} \xi_{\epsilon} \leq \int_{\partial B_{r}} \frac{\partial}{\partial \nu} \Delta u+C\left(\int_{\{\epsilon \leq|x| \leq 2 \epsilon\}} u^{p} \xi_{\epsilon}\right)^{1 / p}
$$

This implies that there exists a constant $C>0$ (independent of $\epsilon$ ) such that

$$
\int_{B_{r}} u^{p} \xi_{\epsilon} \leq C
$$

Now letting $\epsilon \rightarrow 0$, we conclude that $u \in L^{p}\left(B_{r}\right)$.

To show that $u$ is a distribution solution we need to establish 3.1. For any $\varphi \in$ $C_{c}^{\infty}\left(B_{1}\right)$, using $\eta_{\epsilon} \varphi$ as a test function in 1.1 with $\eta_{\epsilon}$ as before gives

$$
\int_{B_{1}} u \Delta^{2}\left(\eta_{\epsilon} \varphi\right)=\int_{B_{1}} u^{p} \eta_{\epsilon} \varphi
$$

By a direct computation, we have

$$
\begin{aligned}
\Delta^{2}\left(\eta_{\epsilon} \varphi\right)= & \eta_{\epsilon} \Delta^{2} \varphi+4 \nabla \eta_{\epsilon} \cdot \nabla \Delta \varphi+2 \Delta \eta_{\epsilon} \Delta \varphi+4 \sum_{i, j=1}^{n}\left(\eta_{\epsilon}\right)_{x_{i} x_{j}} \varphi_{x_{i} x_{i}} \\
& +4 \nabla \Delta \eta_{\epsilon} \cdot \nabla \varphi+\varphi \Delta^{2} \eta_{\epsilon} \\
= & : \eta_{\epsilon} \Delta^{2} \varphi+\psi
\end{aligned}
$$

and by Hölder's inequality, we get

$$
\begin{aligned}
\left|\int_{B_{1}} u \psi\right| & \leq C \epsilon^{-4} \int_{\{\epsilon \leq|x| \leq 2 \epsilon\}} u \\
& \leq C \epsilon^{-4} \cdot \epsilon^{n(1-1 / p)}\left(\int_{\{\epsilon \leq|x| \leq 2 \epsilon\}} u^{p}\right)^{1 / p} \\
& \leq C\left(\int_{\{\epsilon \leq|x| \leq 2 \epsilon\}} u^{p}\right)^{1 / p} \rightarrow 0 \quad \text { as } \epsilon \rightarrow 0 .
\end{aligned}
$$


Letting $\epsilon \rightarrow 0$ in (3.4), then (3.1) follows immediately from the dominated convergence theorem and the proof is complete.

Now we prove that if $u$ is a nonnegative solution of 1.1 in $\mathbb{R}^{n} \backslash\{0\}$, then the sign condition

$$
-\Delta u \geq 0 \quad \text { in } \mathbb{R}^{n} \backslash\{0\}
$$

holds. This allows us to use the maximum principle for $u$ in $\mathbb{R}^{n} \backslash\{0\}$.

Lemma 3.2. Assume $\frac{n}{n-4}<p<\frac{n+4}{n-4}$ and that $u \in C^{4}\left(\mathbb{R}^{n} \backslash\{0\}\right)$ is a nonnegative solution of

$$
\Delta^{2} u=u^{p} \quad \text { in } \mathbb{R}^{n} \backslash\{0\} .
$$

Then $-\Delta u$ is a superharmonic function in $\mathbb{R}^{n}$ in the distributional sense. Moreover,

$$
-\Delta u \geq 0 \quad \text { in } \mathbb{R}^{n} \backslash\{0\} .
$$

Proof. By Lemma 3.1, we have $u \in L_{l o c}^{p}\left(\mathbb{R}^{n}\right)$. Let $\varphi \in C_{c}^{\infty}\left(\mathbb{R}^{n}\right)$ be a nonnegative function. We will prove that

$$
\int_{\mathbb{R}^{n}} \Delta u \Delta \varphi \geq 0
$$

Let $\eta_{\epsilon} \in C^{\infty}\left(\mathbb{R}^{n}\right)$ satisfy (3.2) and 3.3. Multiplying 3.5 by $\eta_{\epsilon} \varphi$ and integrating by parts, we obtain

$$
\begin{aligned}
0 & \leq \int_{\mathbb{R}^{n}} \eta_{\epsilon} \varphi u^{p} \\
& =\int_{\mathbb{R}^{n}} \Delta\left(\eta_{\epsilon} \varphi\right) \Delta u \\
& =\int_{\mathbb{R}^{n}} \Delta u\left(\Delta \varphi \eta_{\epsilon}+2 \nabla \varphi \cdot \nabla \eta_{\epsilon}+\varphi \Delta \eta_{\epsilon}\right) .
\end{aligned}
$$

Denote $\psi=2 \nabla \varphi \cdot \nabla \eta_{\epsilon}+\varphi \Delta \eta_{\epsilon}$. Then $\psi(x) \equiv 0$ for $|x| \leq \epsilon$ and for $|x| \geq 2 \epsilon$, and

$$
|\Delta \psi(x)| \leq C \epsilon^{-4}
$$

Since $n-4-\frac{n}{p}>0$, we have

$$
\begin{aligned}
\left|\int_{\mathbb{R}^{n}} \Delta u \psi\right| & \leq \int_{\mathbb{R}^{n}} u|\Delta \psi| \\
& \leq C \epsilon^{-4}\left(\int_{\{\epsilon \leq|x| \leq 2 \epsilon\}} u^{p}\right)^{1 / p} \epsilon^{n(1-1 / p)} \\
& \leq C \epsilon^{n-4-\frac{n}{p}} \rightarrow 0, \quad \text { as } \epsilon \rightarrow 0 .
\end{aligned}
$$

Therefore, we obtain

$$
\begin{aligned}
\int_{\mathbb{R}^{n}} \Delta u \Delta \varphi & =\lim _{\epsilon \rightarrow 0} \int_{\mathbb{R}^{n}} \Delta u\left(\Delta \varphi \eta_{\epsilon}+2 \nabla \varphi \cdot \nabla \eta_{\epsilon}+\varphi \Delta \eta_{\epsilon}\right) \\
& =\int_{\mathbb{R}^{n}} \varphi u^{p} \geq 0
\end{aligned}
$$


Thus, $-\Delta u$ is a superharmonic function in $\mathbb{R}^{n}$ in the distributional sense.

Let $v_{\epsilon}:=-\Delta u+\epsilon$ for $\epsilon>0$. By Corollary 2.2, we have $\lim _{|x| \rightarrow \infty}|\Delta u(x)|=0$. Therefore, for any $\epsilon>0$, there exists $R_{\epsilon}$ such that

$$
v_{\epsilon}>\frac{\epsilon}{2} \quad \text { for }|x| \geq R_{\epsilon} .
$$

Since $v_{\epsilon}$ is also a superharmonic function in $\mathbb{R}^{n}$ in the distributional sense, we obtain

$$
v_{\epsilon} \geq 0 \quad \text { in } \mathbb{R}^{n} \backslash\{0\} .
$$

Letting $\epsilon \rightarrow 0$, we get $-\Delta u \geq 0$ in $\mathbb{R}^{n} \backslash\{0\}$. This completes the proof.

Let $u$ be a nonnegative solution of 1.1. We use the following transformation of (1.1) (also known as Emden-Fowler transformation): set

$$
t=\ln |x|, \quad \theta=\frac{x}{|x|}
$$

and

$$
w(t, \theta)=|x|^{\frac{4}{p-1}} u(|x|, \theta)=e^{4 t /(p-1)} u\left(e^{t}, \theta\right), \quad t \in(-\infty, 0), \theta \in \mathbb{S}^{n-1} .
$$

By a tedious computation we find that equation (1.1) for $u$ is equivalent to the following equation for $w$ :

$$
\begin{aligned}
\partial_{t}^{(4)} w & +K_{3} \partial_{t}^{(3)} w+K_{2} \partial_{t}^{(2)} w+K_{1} \partial_{t} w+\Delta_{\theta}^{2} w+2 \partial_{t}^{(2)} \Delta_{\theta} w \\
& +K_{3} \partial_{t} \Delta_{\theta} w+J_{1} \Delta_{\theta} w+K_{0} w=w^{p} \quad \text { in }(-\infty, 0) \times \mathbb{S}^{n-1},
\end{aligned}
$$

where $\Delta_{\theta}$ is the Beltrami-Laplace operator on $\mathbb{S}^{n-1}$, the constants $K_{i}=K_{i}(p, n)$ $(i=0, \cdots, 3)$ and $J_{1}=J_{1}(p, n)$ are given by

$$
\begin{aligned}
K_{0}= & \frac{8}{(p-1)^{4}}\left[(n-2)(n-4)(p-1)^{3}+2\left(n^{2}-10 n+20\right)(p-1)^{2}\right. \\
& -16(n-4)(p-1)+32], \\
K_{1}= & -\frac{2}{(p-1)^{3}}\left[(n-2)(n-4)(p-1)^{3}+4\left(n^{2}-10 n+20\right)(p-1)^{2}\right. \\
& -48(n-4)(p-1)+128], \\
K_{2}= & \frac{1}{(p-1)^{2}}\left[\left(n^{2}-10 n+20\right)(p-1)^{2}-24(n-4)(p-1)+96\right], \\
K_{3}= & \frac{2}{p-1}[(n-4)(p-1)-8], \\
J_{1}= & -\frac{2}{(p-1)^{2}}\left[(n-4)(p-1)^{2}+4(n-4)(p-1)-16\right] .
\end{aligned}
$$

Note that if $p<\frac{n+4}{n-4}$, then

$$
(n-4)(p-1)<8 .
$$


It is not difficult to show that

$$
K_{1}=K_{3}=0 \quad \text { if } p=\frac{n+4}{n-4} .
$$

Moreover, we have

Lemma 3.3. Assume $n \geq 5$ and $\frac{n}{n-4}<p<\frac{n+4}{n-4}$. Then

$$
K_{0}>0, \quad K_{1}>0, \quad K_{3}<0 .
$$

Remark 3.1. We emphasize that the sign of $K_{1}$ and $K_{3}$ will be essentially used in our arguments. We also point that $J_{1}<0$ for $\frac{n}{n-4}<p<\frac{n+4}{n-4}$ and the sign of $K_{2}$ depends on $p$ and $n$.

Proof. By [3.9), we easily obtain $K_{3}<0$. Next we will prove that $K_{1}>0$ under the assumptions. For this purpose, we consider the function

$$
f(s)=(n-2)(n-4) s^{3}+4\left(n^{2}-10 n+20\right) s^{2}-48(n-4) s+128
$$

with $s \in\left(\frac{4}{n-4}, \frac{8}{n-4}\right)$. Then

$$
f^{\prime}(s)=3(n-2)(n-4) s^{2}+8\left(n^{2}-10 n+20\right) s-48(n-4) .
$$

Since $f^{\prime}(0)<0, f^{\prime}$ has only one positive root, we denote it by $s_{+}$. We also denote

$$
s_{0}=\frac{4}{n-4} \quad \text { and } \quad s_{1}=\frac{8}{n-4} .
$$

By a direct calculation, we have $f^{\prime}\left(s_{1}\right)=\frac{16\left(n^{2}-4 n+8\right)}{n-4}>0$. Hence we must have $s_{+}<s_{1}$. We consider separately the case $s_{0} \geq s_{+}$and the case $s_{0}<s_{+}$.

Case 1: $s_{0} \geq s_{+}$. In this case we have $f^{\prime}(s)>0$ for all $s \in\left(s_{0}, s_{1}\right)$. By (3.10),

$$
f(s)<f\left(s_{1}\right)=0 \quad \text { for any } s \in\left(s_{0}, s_{1}\right) .
$$

Case 2: $s_{0}<s_{+}$. In this case we have $f^{\prime}(s)<0$ in $\left(s_{0}, s_{+}\right)$and $f^{\prime}(s)>0$ in $\left(s_{+}, s_{1}\right)$. Combining (3.10) and the basic fact $f\left(s_{0}\right)=-\frac{64(n-2)}{(n-4)^{2}}<0$, we obtain

$$
f(s)<\max \left\{f\left(s_{0}\right), f\left(s_{1}\right)\right\}=0 \quad \text { for any } s \in\left(s_{0}, s_{1}\right) .
$$

From these we easily get $K_{1}>0$ if $\frac{n}{n-4}<p<\frac{n+4}{n-4}$.

Now we check $K_{0}>0$. Similarly, we consider

$$
g(s)=(n-2)(n-4) s^{3}+2\left(n^{2}-10 n+20\right) s^{2}-16(n-4) s+32
$$

with $s>\frac{4}{n-4}$. Then

$$
g^{\prime}(s)=3(n-2)(n-4) s^{2}+4\left(n^{2}-10 n+20\right) s-16(n-4) .
$$

Direct calculations show that $g^{\prime}\left(s_{0}\right)=\frac{16(n-2)}{n-4}>0$ and $g\left(s_{0}\right)=0$. From this we get $g^{\prime}(s)>0$ for all $s>s_{0}$ and then

$$
g(s)>g\left(s_{0}\right)=0 \quad \text { for any } s>s_{0} .
$$

Hence we have $K_{0}>0$ if $p>\frac{n}{n-4}$. 
Next we will establish an important monotonicity formula. Let $w$ be a nonnegative solution of (3.8). Define

$$
\begin{aligned}
E(t ; w):= & \int_{\mathbb{S}^{n-1}} \partial_{t}^{(3)} w \partial_{t} w-\frac{1}{2} \int_{\mathbb{S}^{n-1}}\left[\left(\partial_{t}^{2} w\right)^{2}-2 K_{3} \partial_{t}^{2} w \partial_{t} w-K_{2}\left(\partial_{t} w\right)^{2}\right] \\
& +\frac{1}{2} \int_{\mathbb{S}^{n-1}}\left[\left|\Delta_{\theta} w\right|^{2}-J_{1}\left|\nabla_{\theta} w\right|^{2}\right]+\frac{K_{0}}{2} \int_{\mathbb{S}^{n-1}} w^{2} \\
& -\frac{1}{p+1} \int_{\mathbb{S}^{n-1}} w^{p+1}-\int_{\mathbb{S}^{n-1}}\left|\partial_{t} \nabla_{\theta} w\right|^{2}
\end{aligned}
$$

Then we have the following

Lemma 3.4. Assume $\frac{n}{n-4}<p<\frac{n+4}{n-4}$ and that $w$ is a nonnegative $C^{4}$ solution of (3.8). Then, $E(r ; w)$ is non-increasing in $t \in(-\infty, 0)$. Furthermore, we have

$$
\frac{d}{d t} E(t ; w)=K_{3} \int_{\mathbb{S}^{n-1}}\left[\left(\partial_{t}^{2} w\right)^{2}+\left|\partial_{t} \nabla_{\theta} w\right|^{2}\right]-K_{1} \int_{\mathbb{S}^{n-1}}\left(\partial_{t} w\right)^{2} .
$$

Remark 3.2. An analogous monotonicity formula has been derived by the author and Zou [33] to study isolated singularities for a fractional equation. Ghergu-KimShahgholian [14] also obtained a similar monotonicity formula for a second order semilinear elliptic system with power-law nonlinearity.

Proof. Note that

$$
\begin{aligned}
\partial_{t}^{(4)} w \partial_{t} w & =\partial_{t}\left(\partial_{t}^{(3)} w \partial_{t} w\right)-\partial_{t}^{(3)} w \partial_{t}^{2} w \\
& =\partial_{t}\left(\partial_{t}^{(3)} w \partial_{t} w-\frac{1}{2}\left(\partial_{t}^{2} w\right)^{2}\right), \\
\partial_{t}^{(3)} w \partial_{t} w & =\partial_{t}\left(\partial_{t}^{2} w \partial_{t} w\right)-\left(\partial_{t}^{2} w\right)^{2}, \\
\partial_{t}^{2} w \partial_{t} w & =\frac{1}{2} \partial_{t}\left(\partial_{t} w\right)^{2} .
\end{aligned}
$$

Therefore, multiplying Eq. (3.8) by $\partial_{t} w$ and integrating by parts on $\mathbb{S}^{n-1}$, we get

$$
\frac{d}{d t} E(t ; w)=K_{3} \int_{\mathbb{S}^{n-1}}\left[\left(\partial_{t}^{2} w\right)^{2}+\left|\partial_{t} \nabla_{\theta} w\right|^{2}\right]-K_{1} \int_{\mathbb{S}^{n-1}}\left(\partial_{t} w\right)^{2} .
$$

By Lemma 3.3 , we have $K_{1}>0$ and $K_{3}<0$. Hence $\frac{d}{d t} E(t ; w) \leq 0$ and we finish the proof.

Lemma 3.5. Let $w$ be a nonnegative $C^{4}$ solution of (3.8) with $1<p<\frac{n+4}{n-4}$. Then $w$, $\partial_{t} w, \partial_{t}^{2} w, \partial_{t}^{(3)} w, \Delta_{\theta} w$ and $\left|\nabla_{\theta} w\right|$ are uniformly bounded in $(-\infty,-\ln 2) \times \mathbb{S}^{n-1}$.

Proof. Define

$$
u(x)=|x|^{-\frac{4}{p-1}} w(t, \theta),
$$


where $t=\ln |x|$ and $\theta=\frac{x}{|x|}$. Then $u$ is a nonnegative solution of 1.1. By Lemma 2.1. we know that $w$ is uniformly bounded. By Corollary 2.1 we have

$$
\begin{aligned}
&\left|\partial_{t} w\right|+\left|\nabla_{\theta} w\right| \leq C \sum_{i=0}^{1}|x|^{\frac{4}{p-1}+i}\left|\nabla_{x}^{i} u\right| \leq C, \\
&\left|\partial_{t}^{2} w\right|+\left|\Delta_{\theta} w\right| \leq C \sum_{i=0}^{2}|x|^{\frac{4}{p-1}+i}\left|\nabla_{x}^{i} u\right| \leq C, \\
&\left|\partial_{t}^{(3)} w\right| \leq C \sum_{i=0}^{3}|x|^{\frac{4}{p-1}+i}\left|\nabla_{x}^{i} u\right| \leq C .
\end{aligned}
$$

Thus the desired conclusion follows.

Assume $\frac{n}{n-4}<p<\frac{n+4}{n-4}$, from Lemmas 3.4 and 3.5 we deduce that the limit $\lim _{t \rightarrow-\infty} E(t ; w)$ exists. Let $u$ be a nonnegative solution of 1.1), we define

$$
\widetilde{E}(r ; u):=E(t ; w),
$$

where $t=\ln r$ and $w$ is defined as in (3.7). Then we have

$$
\widetilde{E}(0 ; u):=\lim _{r \rightarrow 0^{+}} \widetilde{E}(r ; u)=\lim _{t \rightarrow-\infty} E(t ; w) .
$$

For any $\lambda>0$, define

$$
u^{\lambda}(x):=\lambda^{\frac{4}{p-1}} u(\lambda x) .
$$

Then $u^{\lambda}$ is also a nonnegative solution of (1.1) in $B_{1 / \lambda} \backslash\{0\}$. Moreover, we have

$$
\begin{aligned}
\widetilde{E}\left(r ; u^{\lambda}\right) & =E(t ; w(\cdot+\ln \lambda, \cdot)) \\
& =E(t+\ln \lambda, w) \\
& =E(\lambda r, u) .
\end{aligned}
$$

That is, we get the following scaling invariance

$$
\widetilde{E}\left(r ; u^{\lambda}\right)=\widetilde{E}(\lambda r ; u) .
$$

Lemma 3.6. Let $u \in C^{4}\left(B_{1} \backslash\{0\}\right)$ be a nonnegative solution of 1 1.1) with $\frac{n}{n-4}<p<$ $\frac{n+4}{n-4}$. Then either

$$
\lim _{|x| \rightarrow 0}|x|^{\frac{4}{p-1}} u(x)=0
$$

or

$$
\lim _{|x| \rightarrow 0}|x|^{\frac{4}{p-1}} u(x)=K_{0}^{\frac{1}{p-1}},
$$

where $K_{0}$ is given by (1.4). 
Proof. First we compute the possible values of $\widetilde{E}(0 ; u)$. By Lemma 2.1, $u^{\lambda}$ are uniformly bounded in $C^{4, \alpha}(K)$ on every compact set $K \subset B_{1 / 2 \lambda} \backslash\{0\}$, with some $0<$ $\alpha<1$. Therefore, there exists a nonnegative function $u^{0} \in C^{4}\left(\mathbb{R}^{n} \backslash\{0\}\right)$, such that up to a subsequence of $\lambda \rightarrow 0, u^{\lambda}$ converges to $u^{0}$ in $C_{l o c}^{4}\left(\mathbb{R}^{n} \backslash\{0\}\right)$. Further, $u^{0}$ satisfies

$$
\Delta^{2} u=u^{p} \quad \text { in } \mathbb{R}^{n} \backslash\{0\} .
$$

By Lemma 3.2, we have $-\Delta u^{0} \geq 0$ in $\mathbb{R}^{n} \backslash\{0\}$. The maximum principle gives that either

$$
u^{0} \equiv 0 \quad \text { in } \mathbb{R}^{n} \backslash\{0\}
$$

or

$$
u^{0}>0 \quad \text { in } \mathbb{R}^{n} \backslash\{0\} .
$$

Therefore, by Theorem 4.2 in [24], $u^{0}$ is radially symmetric with respect to the origin 0 . Moreover, by the scaling invariance of $\widetilde{E}$, we have for any $r>0$ that

$$
\widetilde{E}\left(r ; u^{0}\right)=\lim _{\lambda \rightarrow 0} \widetilde{E}\left(r ; u^{\lambda}\right)=\lim _{\lambda \rightarrow 0} \widetilde{E}(\lambda r ; u)=\widetilde{E}(0 ; u) .
$$

Let

$$
w^{0}(t):=|x|^{\frac{4}{p-1}} u^{0}(|x|), \quad t=\ln |x|
$$

Then $w^{0}$ satisfies

$$
\frac{d^{4}}{d t^{4}} w^{0}+K_{3} \frac{d^{3}}{d t^{3}} w^{0}+K_{2} \frac{d^{2}}{d t^{2}} w^{0}+K_{1} \frac{d}{d t} w^{0}+K_{0} w^{0}=\left(w^{0}\right)^{p} \quad \text { in } \mathbb{R} .
$$

From $3.15, E\left(t ; w^{0}\right)=\widetilde{E}\left(r ; u^{0}\right)$ is a constant. By Lemma 3.4,

$$
\frac{d}{d t} E\left(t ; w^{0}\right)=\left|\mathbb{S}^{n-1}\right|\left[K_{3}\left(\frac{d^{2}}{d t^{2}} w^{0}\right)^{2}-K_{1}\left(\frac{d}{d t} w^{0}\right)^{2}\right] \equiv 0 .
$$

Since $K_{3}<0$ and $K_{1}>0$, we get that $\frac{d}{d t} w^{0} \equiv 0$ in $\mathbb{R}$ and then $w^{0}$ is a constant. By (3.16), either $w^{0}=0$ or $w^{0}=K_{0}^{\frac{1}{p-1}}$. Hence, by (3.15) we obtain

$$
\widetilde{E}(0 ; u) \in\left\{0,\left(\frac{1}{2}-\frac{1}{p+1}\right) K_{0}^{\frac{p+1}{p-1}}\left|\mathbb{S}^{n-1}\right|\right\} .
$$

If $\widetilde{E}(0 ; u)=0$, then $u^{0} \equiv 0$. Since this function $u^{0}$ is unique, we conclude that $u^{\lambda} \rightarrow 0$ for any sequence of $\lambda \rightarrow 0$, in $C^{4}(K)$ on every compact set $K \subset \mathbb{R}^{n} \backslash\{0\}$. Therefore, we easily get

$$
\lim _{|x| \rightarrow 0}|x|^{\frac{4}{p-1}} u(x)=0 .
$$

$$
\begin{aligned}
& \text { If } \widetilde{E}(0 ; u)=\left(\frac{1}{2}-\frac{1}{p+1}\right) K_{0}^{\frac{p+1}{p-1}}\left|\mathbb{S}^{n-1}\right| \text {, then we have } \\
& u^{0}(x) \equiv K_{0}^{\frac{1}{p-1}}|x|^{-\frac{4}{p-1}}
\end{aligned}
$$


In this case the function $u^{0}$ is also unique, so we obtain that $u^{\lambda} \rightarrow K_{0}^{\frac{1}{p-1}}|x|^{-\frac{4}{p-1}}$ for any sequence of $\lambda \rightarrow 0$, in $C^{4}(K)$ on every compact set $K \subset \mathbb{R}^{n} \backslash\{0\}$. In particular, we have

$$
u^{\lambda}(x) \rightarrow K_{0}^{\frac{1}{p-1}} \quad \text { as } \lambda \rightarrow 0
$$

in $C\left(\mathbb{S}^{n-1}\right)$. We quickly get

$$
\lim _{|x| \rightarrow 0}|x|^{\frac{4}{p-1}} u(x)=K_{0}^{\frac{1}{p-1}} .
$$

This completes the proof.

Lemma 3.7. Assume $\frac{n}{n-4}<p<\frac{n+4}{n-4}$ and that $u \in C^{4}\left(B_{1} \backslash\{0\}\right)$ is a nonnegative solution of 1.1. If

$$
\lim _{|x| \rightarrow 0}|x|^{\frac{4}{p-1}} u(x)=0
$$

then

$$
\int_{\{|x| \leq 1 / 2\}} u^{(p-1) n / 4}<+\infty
$$

Proof. Set

$$
\varphi(x)=|x|^{-\frac{n-4}{p-1}\left(p-\frac{n}{n-4}\right)} .
$$

We recall that in radial coordinates $r=|x|$, we have

$$
\begin{aligned}
\Delta^{2} \varphi(r)= & \varphi^{(4)}(r)+\frac{2(n-1)}{r} \varphi^{(3)}(r)+\frac{(n-1)(n-3)}{r^{2}} \varphi^{\prime \prime}(r) \\
& -\frac{(n-1)(n-3)}{r^{3}} \varphi^{\prime}(r)
\end{aligned}
$$

Denote

$$
\gamma:=-\frac{n-4}{p-1}\left(p-\frac{n}{n-4}\right)<0 .
$$

Direct calculations show that

$$
\begin{aligned}
\Delta^{2} \varphi= & {[\gamma(\gamma-1)(\gamma-2)(\gamma-3)+2 \gamma(n-1)(\gamma-1)(\gamma-2)} \\
& +\gamma(n-1)(n-3)(\gamma-1)-\gamma(n-1)(n-3)] r^{\gamma-4} \\
= & \gamma(\gamma-2)[(\gamma-1)(\gamma-3)+2(n-1)(\gamma-1)+(n-1)(n-3)] r^{\gamma-4} \\
= & \gamma(\gamma-2)[(\gamma-1)(\gamma+n-4)+(n-1)(\gamma+n-4)] r^{\gamma-4} \\
= & \gamma(\gamma-2)(\gamma+n-2)(\gamma+n-4) r^{\gamma-4} .
\end{aligned}
$$

Since $\gamma+n-4=\frac{4}{p-1}>0$, we have

$$
A:=\gamma(\gamma-2)(\gamma+n-2)(\gamma+n-4)>0 .
$$


That is, we obtain

$$
\frac{\Delta^{2} \varphi}{\varphi}=\frac{A}{|x|^{4}} \quad \text { in } \mathbb{R}^{n} \backslash\{0\} .
$$

For small $\epsilon>0$, let $\zeta_{\epsilon}$ be a smooth cut-off function satisfying

$$
\zeta_{\epsilon}(x)= \begin{cases}1 & \text { for } \epsilon \leq|x| \leq \frac{1}{2} \\ 0 & \text { for }|x| \leq \frac{\epsilon}{2},|x| \geq \frac{3}{4}\end{cases}
$$

and

$$
\left|\nabla^{k} \zeta_{\epsilon}(x)\right| \leq C \epsilon^{-k} \quad \text { for } k=1,2,3,4
$$

Using $\zeta_{\epsilon} \varphi$ as a test function in (1.1) and integrating by parts we obtain

$$
\int_{B_{1}} \zeta_{\epsilon} u \varphi\left(\frac{\Delta^{2} \varphi}{\varphi}-u^{p-1}\right)=-\int_{B_{1}} u F\left(\zeta_{\epsilon}, \varphi\right),
$$

where

$$
\begin{aligned}
F\left(\zeta_{\epsilon}, \varphi\right)= & 4 \nabla \zeta_{\epsilon} \cdot \nabla \Delta \varphi+2 \Delta \zeta_{\epsilon} \Delta \varphi+4 \sum_{i, j=1}^{n}\left(\zeta_{\epsilon}\right)_{x_{i} x_{j}} \varphi_{x_{i} x_{i}} \\
& +4 \nabla \Delta \zeta_{\epsilon} \cdot \nabla \varphi+\varphi \Delta^{2} \zeta_{\epsilon} .
\end{aligned}
$$

By 3.21, 3.22 and Lemma 2.1, we estimate

$$
\begin{aligned}
\left|\int_{B_{1}} u F\left(\zeta_{\epsilon}, \varphi\right)\right| \leq & \left|\int_{\left\{\frac{1}{2} \leq|x| \leq \frac{3}{4}\right\}} u F\left(\zeta_{\epsilon}, \varphi\right)\right|+\left|\int_{\left\{\frac{\epsilon}{2} \leq|x| \leq \epsilon\right\}} u F\left(\zeta_{\epsilon}, \varphi\right)\right| \\
\leq & C_{1}+C_{2} \int_{\left\{\frac{\epsilon}{2} \leq|x| \leq \epsilon\right\}} u\left[\frac{1}{\epsilon}|x|^{\gamma-3}+\frac{1}{\epsilon^{2}}|x|^{\gamma-2}\right. \\
& \left.+\frac{1}{\epsilon^{3}}|x|^{\gamma-1}+\frac{1}{\epsilon^{4}}|x|^{\gamma}\right] \\
\leq & C_{1}+C_{2} \epsilon^{n} \epsilon^{\gamma-4} \epsilon^{-\frac{4}{p-1}} \leq C_{1}+C_{2}<+\infty
\end{aligned}
$$

where $C_{1}=C_{1}(p, n, u)$ and $C_{2}=C_{2}(p, n)$ are two positive constants (independent of $\epsilon$ ). Hence

$$
\int_{B_{1}} \zeta_{\epsilon} u \varphi\left(\frac{\Delta^{2} \varphi}{\varphi}-u^{p-1}\right) \leq C_{2}<+\infty
$$

uniformly in $\epsilon$. By the assumption 3.17,

$$
u^{p-1}(x)=o(1)|x|^{-4} \quad \text { as }|x| \rightarrow 0 .
$$

This together with (3.20) and 3.24) gives

$$
\int_{B_{1}} \zeta_{\epsilon} u|x|^{\gamma-4} \leq C_{3}<+\infty
$$


where $C_{3}$ is a positive constant independent of $\epsilon$. Therefore, by Lemma 2.1,

$$
\begin{aligned}
\int_{\left\{\epsilon \leq|x| \leq \frac{1}{2}\right\}} u^{(p-1) n / 4} & =\int_{\left\{\epsilon \leq|x| \leq \frac{1}{2}\right\}} u u^{((p-1) n-4) / 4} \\
& \leq C(p, n) \int_{\left\{\epsilon \leq|x| \leq \frac{1}{2}\right\}} u|x|^{-((p-1) n-4) /(p-1)} \\
& =C(p, n) \int_{\left\{\epsilon \leq|x| \leq \frac{1}{2}\right\}} u|x|^{\gamma-4} \\
& \leq C(p, n) \int_{B_{1}} \zeta_{\epsilon} u|x|^{\gamma-4} \leq C(p, n) C_{3}<+\infty
\end{aligned}
$$

Letting $\epsilon \rightarrow 0$, we get $(3.18)$ by the dominated convergence theorem.

Now we give a new method to obtain the removable singularity theorem. For our fourth order equation (1.1), the classical methods based on the maximum principle to second order problems (such as see [1, 4, 8, 22]) fail. We remark that our method also apply to higher order equations. This method is based on the following Regularity Lifting Theorem from Chen-Li [9].

Let $V$ be a Hausdorff topological vector space. Suppose there are two extended norms (i.e., the norm of an element in $V$ might be infinity) defined on $V$,

$$
\|\cdot\|_{X},\|\cdot\|_{Y}: V \rightarrow[0,+\infty]
$$

Let

$$
X:=\left\{v \in V:\|v\|_{X}<+\infty\right\} \quad \text { and } \quad Y:=\left\{v \in V:\|v\|_{Y}<+\infty\right\} .
$$

Assume that spaces $X$ and $Y$ are complete under the corresponding norms and the convergence in $X$ or in $Y$ implies the convergence in $V$.

Theorem 3.1. ( [9], Theorem 3.3.1) Let $T$ be a contraction map from $X$ into itself and from $Y$ into itself. Assume that $f \in X$ and that there exists a function $g \in Z:=X \cap Y$ such that $f=T f+g$ in $X$. Then $f$ also belongs to $Z$.

Remark 3.3. We usually choose $V$ to be the space of distributions, and $X$ and $Y$ to be function spaces, for instance, $X=L^{p}$ and $Y=W^{1, q}$.

Next we will use this Regularity Lifting Theorem to prove a removable singularity result.

Lemma 3.8. Assume $\frac{n}{n-4}<p<\frac{n+4}{n-4}$ and that $u \in C^{4}\left(B_{1} \backslash\{0\}\right)$ is a nonnegative solution of (1.1). If

$$
\int_{\{|x| \leq 1 / 2\}} u^{(p-1) n / 4}<+\infty
$$

then the singularity at $x=0$ is removable, i.e., $u(x)$ can be extended to a $C^{4}$ solution of (1.1) in the entire ball $B_{1}$. 
Remark 3.4. For the second order equation (1.2), a similar result for removable singularity was proved by Gidas-Spruck [15]. However, their proof is based on a double application of the De Giorgi-Nash-Moser bootstrap arguments, which cannot be applied to our fourth order problem (1.1).

Proof. Let $G_{2}(x, y)$ be the Green's function of $\Delta^{2}$ in $B_{1 / 2}$ with homogeneous Dirichlet boundary conditions, Then, for each fixed $y \in B_{1}, G_{2}(\cdot, y)$ is a distributional solution of

$$
\begin{cases}\Delta^{2} G_{2}(\cdot, y)=\delta(\cdot-y) & \text { in } B_{1 / 2}, \\ G_{2}(\cdot, y)=\frac{\partial G_{2}(\cdot, y)}{\partial \nu}=0 & \text { on } \partial B_{1 / 2},\end{cases}
$$

and there exists positive constant $C_{n}$ such that

$$
0<G_{2}(x, y) \leq \Gamma_{2}(|x-y|):=C_{n}|x-y|^{4-n} \quad \text { for } x, y \in B_{1 / 2}, x \neq y .
$$

Define

$$
v(x):=-u(x)+\int_{B_{1 / 2}} G_{2}(x, y) u^{p}(y) d y, \quad x \in B_{1 / 2},
$$

then $v \in L^{1}\left(B_{1 / 2}\right)$. Moreover, by Lemma 3.1, $v$ satisfies

$$
\Delta^{2} v=0 \quad \text { in } B_{1 / 2}
$$

in the distributional sense. Using Theorem 7.23 in [27], we get $v \in L_{l o c}^{\infty}\left(B_{1 / 2}\right)$.

Now we split the right hand side of 1.1 into two parts:

$$
u^{p}=u^{p-1} u:=a(x) u .
$$

Then, by the assumption [3.25], $a(x) \in L^{\frac{n}{4}}\left(B_{1 / 2}\right)$. For any positive number $L>0$, let

$$
a_{L}(x)= \begin{cases}a(x) & \text { if }|a(x)| \geq L, \\ 0 & \text { otherwise }\end{cases}
$$

and

$$
a_{M}(x)=a(x)-a_{L}(x) .
$$

Define the linear operator

$$
\left(T_{L} w\right)(x)=\int_{B_{1 / 4}} G_{2}(x, y) a_{L}(y) w(y) d y .
$$

Then $u$ satisfies the equation

$$
u(x)=\left(T_{L} u\right)(x)+F_{L}(x) \quad \text { in } B_{1 / 4},
$$

where

$$
F_{L}(x)=\int_{B_{1 / 4}} G_{2}(x, y) a_{M}(y) u(y) d y-v(x)+h(x)
$$


and

$$
h(x)=\int_{\left\{\frac{1}{4} \leq|y| \leq \frac{1}{2}\right\}} G_{2}(x, y) u^{p}(y) d y .
$$

Note that

$$
\begin{aligned}
|h(x)| & \leq C \int_{\left\{\frac{1}{4} \leq|y| \leq \frac{1}{2}\right\}} G_{2}(x, y) d y \leq C \int_{\left\{\frac{1}{4} \leq|y| \leq \frac{1}{2}\right\}}|x-y|^{4-n} d y \\
& \leq C \int_{B_{1}}|y|^{4-n} d y \leq C \quad \text { for all } x \in B_{1 / 4} .
\end{aligned}
$$

Hence $v, h \in L^{\infty}\left(B_{1 / 4}\right)$.

We will prove that, for any $\frac{n}{n-4}<q<\infty$,

(1) $T_{L}$ is a contracting operator from $L^{q}\left(B_{1 / 4}\right)$ to $L^{q}\left(B_{1 / 4}\right)$ for $L$ large.

(2) $F_{L} \in L^{q}\left(B_{1 / 4}\right)$.

Then, by the Regularity Lifting Theorem 3.1 we obtain $u \in L^{q}\left(B_{1 / 4}\right)$ for any $\frac{n}{n-4}<q<\infty$.

(1) The estimate of the operator $T_{L}$.

For any $\frac{n}{n-4}<q<\infty$, there exists $1<r<\frac{n}{4}$ such that

$$
\frac{1}{q}=\frac{1}{r}-\frac{4}{n}
$$

By Hardy-Littlewood-Sobolev inequality and Hölder inequality, we have

$$
\begin{aligned}
\left\|T_{L} w\right\|_{L^{q}\left(B_{1 / 4}\right)} & \leq\left\|\Gamma_{2} * a_{L} w\right\|_{L^{q}\left(\mathbb{R}^{n}\right)} \leq C\left\|a_{L} w\right\|_{L^{r}\left(B_{1 / 4}\right)} \\
& \leq\left\|a_{L}\right\|_{L^{\frac{n}{4}}\left(B_{1 / 4}\right)}\|w\|_{L^{q}\left(B_{1 / 4}\right)} .
\end{aligned}
$$

Since $a(x) \in L^{\frac{n}{4}}\left(B_{1 / 4}\right)$, we can choose $L$ sufficiently large, such that

$$
\left\|a_{L}\right\|_{L^{\frac{n}{4}}\left(B_{1 / 4}\right)} \leq \frac{1}{2}
$$

Therefore, $T_{L}: L^{q}\left(B_{1 / 4}\right) \rightarrow L^{q}\left(B_{1 / 4}\right)$ is a contracting operator for $L$ large.

(2) The integrability of the function $F_{L}(x)$.

Obviously, we only need to show that, for any $\frac{n}{n-4}<q<\infty$,

$$
F_{L}^{1}(x):=\int_{B_{1 / 4}} G_{2}(x, y) a_{M}(y) u(y) d y \in L^{q}\left(B_{1 / 4}\right) .
$$

Since $a_{M}(x)$ is a bounded function, we have

$$
\left\|F_{L}^{1}\right\|_{L^{q}\left(B_{1 / 4}\right)} \leq\left\|a_{M} u\right\|_{L^{r}\left(B_{1 / 4}\right)} \leq C\|u\|_{L^{r}\left(B_{1 / 4}\right)} .
$$


By the assumption (3.25), $u \in L^{r}\left(B_{1 / 4}\right)$ for any $1<r \leq \frac{(p-1) n}{4}$. Note that

$$
q=\frac{(p-1) n}{4(2-p)} \quad \text { if } r=\frac{(p-1) n}{4} .
$$

Hence, we conclude that, for the following values of $q$,

$$
\begin{cases}1<q<\infty & \text { if } p \geq 2 \\ 1<q \leq \frac{(p-1) n}{4(2-p)} & \text { if } p<2\end{cases}
$$

$F_{L}(x) \in L^{q}\left(B_{1 / 4}\right)$.

Using the Regularity Lifting Theorem 3.1, we obtain

$$
\begin{cases}u \in L^{q}\left(B_{1 / 4}\right) & \text { for any } 1<q<\infty \quad \text { if } p \geq 2 \\ u \in L^{q}\left(B_{1 / 4}\right) & \text { for any } 1<q \leq \frac{(p-1) n}{4(2-p)} \quad \text { if } p<2 .\end{cases}
$$

Now we note that from the starting point where $u \in L^{\frac{(p-1) n}{4}}\left(B_{1 / 4}\right)$, we get

$$
u \in L^{r}\left(B_{1 / 4}\right) \quad \text { with } r=\frac{(p-1) n}{4(2-p)}, \quad p<2 .
$$

By a similar argument as above, we get

$$
\begin{cases}u \in L^{q}\left(B_{1 / 4}\right) & \text { for any } 1<q<\infty \quad \text { if } p \geq \frac{3}{2} \\ u \in L^{q}\left(B_{1 / 4}\right) & \text { for any } 1<q \leq \frac{(p-1) n}{4(3-2 p)} \quad \text { if } p<\frac{3}{2} .\end{cases}
$$

Hence by iteration we have for $k=1,2, \cdots$,

$$
\begin{cases}u \in L^{q}\left(B_{1 / 4}\right) & \text { for any } 1<q<\infty \quad \text { if } p \geq \frac{k+1}{k}, \\ u \in L^{q}\left(B_{1 / 4}\right) & \text { for any } 1<q \leq \frac{(p-1) n}{4[(k+1)-k p]} \quad \text { if } p<\frac{k+1}{k} .\end{cases}
$$

This implies that for any fixed dimension $n$, a finite number of iterations gives

$$
u \in L^{q}\left(B_{1 / 4}\right) \quad \text { for any } 1<q<\infty .
$$

Finally, By Hölder inequality, we have

$$
\int_{B_{1 / 4}} G_{2}(x, y) u^{p}(y) d y \in L^{\infty}\left(B_{1 / 4}\right),
$$

From this and (3.26) we easily deduce that $u \in L^{\infty}\left(B_{1 / 4}\right)$. By estimates of elliptic equations, $u(x)$ is smooth at 0 . Therefore 0 is a removable singularity.

Proof of Theorem 1.1 The proof of Theorem 1.1 is now just a combination of Lemmas 3.6, 3.7 and 3.8. 
Acknowledgments. The author would like to thank Professor Sun-Yung A. Chang for many helpful discussions and comments. The author would also like to thank his advisor Professor Wenming Zou for his constant support and encouragement. This work was done during the author's visit to Princeton University. He thanks Tsinghua University for funding his visit and thanks the Department of Mathematics at Princeton University for kind hospitality.

\section{References}

[1] P. Aviles, Local behavior of solutions of some elliptic equations, Comm. Math. Phys., 108 (1987) 177-192.

[2] M.-F. Bidaut-Véron, L. Véron, Nonlinear elliptic equations on compact Riemannian manifolds and asymptotics of Emden equations, Invent. Math., 106 (3) (1991) 489-539.

[3] L. Caffarelli, B. Gidas, J. Spruck, Asymptotic symmetry and local behavior of semilinear elliptic equations with critical Sobolev growth, Comm. Pure Appl. Math., 42 (1989) 271-297.

[4] L. Caffarelli, T. Jin, Y. Sire, J. Xiong, Local analysis of solutions of fractional semi-linear elliptic equations with isolated singularities, Arch. Ration. Mech. Anal., 213 (1) (2014) 245-268.

[5] G. Caristi, E. Mitidieri, Harnack inequality and applications to solutions of biharmonic equations, Operator Theory: Advances and Applications, 168 (2006) $1-26$.

[6] S.-Y. A. Chang, P. C. Yang, On uniqueness of solutions of $n$th order differential equations in conformal geometry, Math. Res. Lett., 4 (1997) 91-102.

[7] C.-C. Chen, C.-S. Lin, Estimates of the conformal scalar curvature equation via the method of moving planes, Comm. Pure Appl. Math., 50 (1997) 971-1071.

[8] C.-C. Chen, C.-S. Lin, On the asymptotic symmetry of singular solutions of the scalar curvature equations, Math. Ann., 313 (1999) 229-245.

[9] W. Chen, C. Li, Methods on nonlinear elliptic equations, AIMS Ser. Differ. Equ. Dyn. Syst., vol. 4, 2010.

[10] A. Ferrero, H.-C. Grunau, The Dirichlet problem for supercritical biharmonic equations with power-type nonlinearity, J. Differential Equations, 234 (2007) 582-606.

[11] R. L. Frank, T. König, Classification of positive singular solutions to a nonlinear biharmonic equation with critical exponent, Anal. PDE, 12, No. 4 (2019) 11011113.

[12] F. Gazzola, H.-C. Grunau, Radial entire solutions for supercritical biharmonic equations, Math. Ann., 334 (2006) 905-936.

[13] F. Gazzola, H.-C. Grunau, G. Sweers, Polyharmonic boundary value problems: positivity preserving and nonlinear higher order elliptic equations in bounded domains. In: Lecture Notes in Mathematics, issue no. 1991. Springer, Berlin (2010) 
[14] M. Ghergu, S. Kim, H. Shahgholian, Isolated singularities for semilinear elliptic systems with power-law nonlinearity, arXiv:1804.04291.

[15] B. Gidas, J. Spruck, Global and local behavior of positive solutions of nonlinear elliptic equations, Comm. Pure Appl. Math., 34 (1981) 525-598.

[16] M.d.M. González, Classification of singularities for a subcritical fully nonlinear problem, Pacific J. Math., 226 (2006) 83-102.

[17] Z. Guo, J. Wei, Qualitative properties of entire radial solutions for a biharmonic equation with supcritical nonlinearity, Proc. Amer. Math. Soc., 138 (2010) 39573964.

[18] Z. Guo, J. Wei, F. Zhou, Singular radial entire solutions and weak solutions with prescribed singular set for a biharmonic equation, J. Differential Equations, 263 (2017) 1188-1224.

[19] Z.-C. Han, Y.Y. Li, E.V. Teixeira, Asymptotic behavior of solutions to the $\sigma_{k^{-}}$ Yamabe equation near isolated singularities, Invent. Math., 182 (2010) 635-684.

[20] F. Hang, P. C. Yang, Lectures on the fourth-order $Q$ curvature equation, pp. 133 in Geometric analysis around scalar curvatures, edited by F. Han et al., Lect. Notes Ser. Inst. Math. Sci. Natl. Univ. Singap. 31, World Scientific, Hackensack, NJ, 2016.

[21] T. Jin, J. Xiong, Asymptotic symmetry and local behavior of solutions of higher order conformally invariant equations with isolated singularities, arXiv: 1901. $01678 \mathrm{v} 2$.

[22] N. Korevaar, R. Mazzeo, F. Pacard, R. Schoen, Refined asymptotics for constant scalar curvature metrics with isolated singularities, Invent. Math., 135 (2) (1999) 233-272.

[23] Y.Y. Li, Conformally invariant fully nonlinear elliptic equations and isolated singularities, J. Funct. Anal., 233 (2006) 380-425.

[24] C.-S. Lin, A classification of solutions to a conformally invariant equation in $\mathbb{R}^{n}$, Comm. Math. Helv., 73 (1998) 206-231.

[25] P.-L. Lions, Isolated singularities in semilinear problems, J. Differential Equations, 38 (1980) 441-450.

[26] L. Martinazzi, Classification of solutions to the higher order Liouville's equation on $\mathbb{R}^{2 m}$, Math. Z., 263 (2009) 307-329.

[27] D. Mitrea, Distributions, partial differential equations, and harmonic analysis, Springer, New York (2013).

[28] P. Polácik, P. Quittner, P. Souplet, Singularity and decay estimates in superlinear problems via Liouville-type theorems, I: Elliptic equations and systems, Duke Math. J., 139 (2007) 555-579.

[29] R. Soranzo, Isolated singularities of positive solutions of a superlinear biharmonic equation, Potential Analysis, 6 (1997) 57-85.

[30] S. Taliaferro, L. Zhang, Asymptotic symmetries for conformal scalar curvature equations with singularity, Calc. Var. PDE, 26 (2006) 401-428. 
[31] J. Wei, X. Xu, Classification of solutions of high order conformally invariant equations, Math. Ann., 313 (1999) 207-228.

[32] M. Winkler, Refined asymptotics for entire solutions of a biharmonic equation with a supercritical nonlinearity, Math. Ann., 348 (2010) 633-666.

[33] H. Yang, W. Zou, On isolated singularities of fractional semi-linear elliptic equations, arXiv:1804.00817. 